\title{
A New Analytic/Synthetic/Horotic Paradigm
}

From Mathematical Gesture to Synthetic/Horotic Reasoning

Giovanni Maddalena and Fernando Zalamea

\section{CpenEdition}

\section{Journals}

Electronic version

URL: http://journals.openedition.org/ejpap/741

DOI: 10.4000/ejpap.741

ISSN: 2036-4091

Publisher

Associazione Pragma

\section{Electronic reference}

Giovanni Maddalena and Fernando Zalamea, «A New Analytic/Synthetic/Horotic Paradigm », European Journal of Pragmatism and American Philosophy [Online], IV-2 | 2012, Online since 24 December 2012, connection on 01 May 2019. URL : http://journals.openedition.org/ejpap/741 ; DOI : 10.4000/ejpap.741

This text was automatically generated on 1 May 2019.

\section{(c) $(1) \odot$}

Author retains copyright and grants the European Journal of Pragmatism and American Philosophy right of first publication with the work simultaneously licensed under a Creative Commons AttributionNonCommercial-NoDerivatives 4.0 International License. 


\title{
A New Analytic/Synthetic/Horotic Paradigm
}

From Mathematical Gesture to Synthetic/Horotic Reasoning

\author{
Giovanni Maddalena and Fernando Zalamea
}

\section{From Analytics to Synthetics: Kant's Heritage and Peirce's Pragmatism}

Peirce's pragmatism introduces many new philosophical tools - suffice it to mention semiotic, abductive logic, a heuristic based on continuity, scholastic realism - all essential components of a never fully realized broader philosophical project. Peirce usually identified it as 'the truth of continuity' or 'the truth of synechism' (Peirce 1998: 335), or else the method of justifying the 'nature of Sequence,' namely the nature of pragmatism, the rule that enables to know meanings through conceivable effects. But this project required a vast panoptic view necessarily covering different fields, according to a particular order. An ordered list of topics could be the one proposed in 'Prolegomena to an Apology for Pragmaticism' (Peirce 1931-35: vol. 4, § 530-84): continuity, phaneroscopy, signs, existential graphs, kinds of reasoning. In the drafts and the articles of the series written for The Monist (1905-06) he stressed also the role of normative sciences. In that entire series and in the following years he tried in vain to fully explain this or a similar order he had in mind.

2 These efforts were 'in vain' because Peirce tended to lose his track while tilling the 'virgin soil' (Peirce 1931-35: vol. 1, § 128) of those many fields of research he himself discovered. And here a complementary question arises: why did he get lost? Part of the reason is that all the above topics were products of his original insight, and Peirce was eager to explain them precisely. The mathematical definition of continuity stemmed from his thirty years of studying Cantor's set theory, and Peirce had independently discovered Cantor's theorem and Cantor's subsequent paradox. Peirce could not know that Cantor had discovered the same paradox and, in contrast with the German mathematician, he saw 
the immense philosophical impact of this discovery. But in his lectures and in his papers or drafts he spent a lot of time explaining the mathematical basis of the theorem and, after 1900, of the paradox, saving little time and space for their philosophical impact. As a result, his articles and manuscripts often treat either the mathematical explanation alone or the philosophical consequence alone, and it is difficult to put the two together.

Another part of the reason for the lack of a final systematization is that Peirce did not get lost for lack of direction but for failing to get out of his analytic pattern. What does that mean? According to Kant, an analytic judgment subsumes a predicate under a subject, while a synthetic judgment has to look outside the subject-concept into experience, to capture how the predicate is connected to, but not subsumed within, the concept. As Quine, and Kripke after him, stressed, in Kant's work analysis, aprioricity, and necessity form a circular cluster where any element justifies, but also coincides with, the others. Analytic judgments are necessary because they are a priori, and being a priori they are necessary and hence analytic. In this way logic (analysis), epistemic (a priori), and metaphysical (necessary) levels coincide, furnishing the pattern of true or warranted knowledge.

But Kant's aim was not at all the defense of the uniqueness of analytic judgments. He was aware that, as much as analytic judgments are important, they do not bring about acquisition of new knowledge, which is characteristic of synthesis. What is the kind of synthesis Kant was looking for? There is a synthesis which is only the reverse of analysis. This is the operation of combining elements that come from the 'dissection of concepts' produced by analysis. Once we have broken up the concept of the subject, we can work on the elements we found.

5 The other 'synthesis' is the one which gives 'unity.' But when Kant talks about the original synthetic unity of apperception as the kind of combination that analysis presupposes, he qualifies it as 'pure,' not empirical, 'one and the same' (Kant 1781/87, 177, B 132). The unity that this pure apperception creates is 'transcendental' (ibid.), and 'precedes a priori all my determinate thought' (Kant 1781/87, 177, B 135). Synthesis precedes analysis but the character of universality (here conjoined with 'sameness') and aprioricity would shape it according to the analytic pattern, which remains the ideal sample of every knowledge.

6 Summing up, the idea of knowledge is really founded upon an analytic pattern, and that is why Kant seeks to solve the problem of knowledge by using critical, that is, analytic tools only. Kant provides an analytic of synthetic judgments whereby he defines (analytically) the steps through which we arrive at a synthetic representation of reality. Only by breaking down this path step by step is Kant assured of having caught the gist of what knowledge is. The only real synthesis would come up with the critique of judgment, but once again it will have a form that combines (adds to one another) elements of the two previous critiques. In this way Kant simply systematized the long heritage of Western philosophy, but he made it clear that theoretical or pure knowledge is either analytic or something that is analyzable, namely broken down into necessary, a priori pieces.

7 Young Peirce shows no awareness of the complex, presupposed circularity among aprioricity, analyticity, and necessity. But he had enough philosophical insight to sense immediately that something was missing. As early as 1869 , in 'Validity of the Laws of Logic,' he pointed out that far more fundamental than the question of the possibility of synthetic apriori reasoning was that of the possibility of synthetic judgments merely (Peirce 1981-2010: vol. 2, 267-8). Peirce wanted to go farther than his German master in 
criticizing judgments, and to do so he got rid of the a priori principle. A different path of reasoning was needed, and Peirce tried to provide it through both semiotics and the a posteriori method of science. Accordingly, Peirce listed aprioricity as one of the weak ineffective methods for fixing a belief (Peirce 1981-2010: vol. 3, 252-3). The method of science always remained for Peirce an a posteriori method; he spent his life trying to explain and justify the nature of 'ampliative' reasoning, another phrase for synthetic reasoning.

However, when in the 1877-78 Popular Science Monthly series Peirce gave the justification for induction - which at the time he thought included also hypotheses - he surprisingly relied metaphorically on an 'extension' of the a priori principle (Peirce 1981-2010: vol. 3, 304). It is of course a rhetorical expedient, since Peirce's conception of experience had already been forged in the semiotic a posteriori hearth, but it is still a significant episode.

Peirce would then explain that synthetic inference is founded upon the manner of obtaining facts, thus ensuring only the 'degree of trustworthiness of our proceeding' (Peirce 1981-2010: vol. 3, 305). In this way Peirce transforms Kant's a priori principle into a methodological guarantee resting on a certain interpretation of the general statement that 'whatever is universally true is involved in the conditions of experience.' So then does the idea of necessary, universal truth move from Kant's a priori categories to Peirce's methodological conditions. In such a passage, universal analytic truth loses part of its 'necessity' by becoming merely 'trustworthy.' Still, the path Peirce follows remains through the idea of necessity or universality. Like Kant with his schematism, Peirce is after synthetic reasoning but gets to it through the analytic path. The outcome is that ampliative or synthetic arguments do not tend to the necessity of content but to the necessity of method.

In 1903's Lowell Lectures Peirce distinguishes synthetic from analytic judgments, attributing the former to mathematics (hence to necessary reasoning) and the latter to logic, which tries 'to find out how inferences necessary and probable are composed.' Once again, logic is confined to the analytic scheme of composition/decomposition. Therefore, reasoning can be necessary or ampliative, but the logician's work is in both cases to find its composition, confirming that analysis presides over our methodological research even when, as in induction or hypotheses, it does not preside over the concepts at stake.

But in spite of Peirce's analytic project, pragmatism chiefly concerns our concrete synthetic way of thinking. The synthetic process, however, is not the Kantian one that seeks to attain, in both form and content, the kind of clearness that analysis has. All the tools Peirce crafted, from the list of categories to perceptual judgments, from the pragmatic maxim to abduction, from existential graphs to rational instinct, describe a changing synthetic process of thinking rather than the fixed analytic/synthetic reasoning (and judgment) described by Kant. Peirce does not seem to have realized that his tools were hinting towards a completely different path of thought. This tension between the analytic background of his thought and the 'true synthetic' [only 'synthetic' from now on] purpose of those same analyses explains also Peirce's progressive shifts from Kant to Hegel, and his more and more evident epistemic turn in the philosophy of science. This tension shows a different kind of possibility. How can we define synthetic and analytic starting from the change in thought that Peirce was tracking down without seeing the different picture he was formulating? 


\section{A New Paradigm: Analytics, Synthetics, Horotics}

12 We proceed with some new definitions. A synthetic judgment is a judgment that recognizes identity through changes. An analytic judgment is a judgment that loses identity through changes. A horotic judgment (from horos, border) ${ }^{1}$ is a judgment that is blind to identity through changes. In these three definitions one can change 'judgment' by 'reasoning,' opening thus new doors to inquire an extended gnoseological spectrum (for further development of the definitions see Maddalena 2009).

Why do we need a new definition? One of the reasons for this change is required by Peirce's failed attempt to make complete sense of many tools he himself provided. We already described the tension between Peirce's analytic tools and his synthetic project. Beyond that, there are other compelling reasons. One is the clear impasse of analytic philosophy. That kind of philosophy, to which contemporary thought owes so much for its precision and productivity, seems now stuck in a scholasticism full of definitions detached from experiential reality and not really committed to improving our understanding of the world and its transformations. The fact is that analytic philosophy has now come to manifest the incompleteness of the criterion of necessity/analyticity/ aprioricity displayed by Kant. And its critiques, most importantly Quine and Kripke, offered only internal criticisms that failed to change the criterion itself.

The good results of analytic philosophy are due to an efficient understanding of a certain kind of logic (first-order classical logic) based on a certain kind of mathematics (Cantorian set theory). But the 'scholasticism' currently in vogue underlines a limitation of this logic: though analytic logic serves pretty well to split up definitions as called for Kant's heritage, it is intrinsically an endless process. From science to grammar, definitions rarely provide an exact fit: they are always too large or too tight. They are a useful tool but are not the only one, and for sure not the one apt to grasp changes in reality. New mathematics and new kinds of logics have already arisen (see section 5): the time has come to try a new philosophical paradigm.

15 And why do we need this new definition we are proposing? Synthetic reasoning is the original kind that we use in everyday life, and it needs a new paradigm that we will try to expose and justify in the rest of this article. This study does not want to deny, minimize or deconstruct the success of analytic reasoning as it has been carried on so far. We want only to show that analytic reasoning needs and has always used a complementary type of understanding in order to work properly. The possibility of both and the transition between them are the pillars of this different view. We will see that the logical core of synthetic reasoning is both analytically and synthetically describable. Arising from Peirce's studies, this view immediately understands the logical importance of horotics, as the indispensable complement to the two kinds proposed.

The first task in order to show the plausibility of our paradigm is to explain what is change and how we can study it. We recall that Peirce's phenomenology (phaneroscopy in his terminology) postulates the existence of three basic categories in nature and knowledge: firstness (immediacy, possibility, monadicity), secondness (action-reaction, actuality, binarism) and thirdness (mediation, necessity, continuity). We will understand change from a systemic point of view, in the sense that a given in-formation (first) produces a new trans-formation (third) through precise preparation, correlation and creative techniques (seconds) which help to master and modify the initial data. Then, 
given an informational change, an analytic judgment emphasizes differences and thus loses identities of data, focusing on the result of the transformation. On the other hand, a synthetic judgment proposes an integrating view which recognizes fragments of identity, focusing on similar structural processes of the transformation. Finally, a horotic judgment defines a frontier, a border, a sort of pendular differential and integral calculus which focuses on the possibility of relations, that is on the relative spectrum of the transformation, and is thus blind to detect actual identities.

Hetics can, in turn, be approached from three distinct points of view: (i) extrinsically, (ii) intrinsically, (iii) synthetically. Extrinsically (i), horotics can be understood as a completion of the classical dyad analysis/synthesis with respect to Peirce's three categories. Since Greek thought, analysis and synthesis have appeared as complementary polarities (decomposition/composition, part/all, discontinuous/ continuous, element/ structure, etc.), but the intermediate transit between polarities has not been well investigated. In a similar vein to the emergence of generalized quantifiers in Abstract Model Theory à la Lindström, which study intermediate situations between the particular (E) and the general (A) associated to intermediate classes of structures, horotics pretends to open the way to general forms of reasoning beyond the polarity analysis/synthesis. Intrinsically (ii), horotics can be seen as the exact study of the borders of knowledge, merging thus with a strong tradition of critical thinkers akin to the visualization of frontiers and their crossovers: Peirce (logic of continuity), Florenski (borders between science, art and theology), Warburg (seismography of art history), Benjamin (residues in cultural studies), Bakhtin (literary frontiers), Merleau-Ponty (Humanity as a border between Culture and Nature), Blumenberg (evolution of metaphorical images), among many other indispensable xxth century thinkers. Synthetically (iii), horotics achieves its full richness through self-reference and self-regulation processes (frontiers of the frontier, limits of the limit, progressive refinement of borders, etc.) which foster the growth of phenomenological, epistemical and metaphysical orders (topological object, continuous logic, Peirce's synechism, etc.).

In what follows, we will be detailing our general paradigm along three main case studies Peirce's 'mathematical gestures' (section 3), Gödel's synthetic approaches to intuitionistic logic (section 4), forces in play in Contemporary Mathematics (section 5) -, and we will finish prospecting its pertinence (section 6) for the coming years.

\section{The Paradigm Along Peirce's Mathematical Gestures}

19 The triad analytics/synthetics/horotics glues naturally if we use Peirce's fundamental insight: the concept of continuity, the 'keystone' of pragmatism. The study of continuity from a mathematical and a logical standpoint shows how to understand change and invariance. From the very beginning of Peirce's intellectual proposal, continuity is paired with representation, or cognition, becoming increasingly unified with it the more he was discovering the real mathematical structure of continuity. There are many changes in Peirce's mathematical approach to the topic, but substantially they all focus on the proof of Cantor's theorem and paradox that Peirce independently discovered in the late 1890s. With this proof Peirce understood that there is an infinite series of multitudes that Cantor's set theory can reach, but that those multitudes are always bounded to an imperfect or pseudo-continuity that depends on the unavoidable singularity of the initial 
definition of set or collection (Peirce 1976: vol. 3, 774-5). Peirce's definition implies that a collection is an individual whose existence depends on the regularities among other individuals. These 'regularities' can be identified by the characters its members possess (ineunts) or exclude (exeunts) (Peirce 1976: vol. 3, 776). Therefore, if a collection implies by definition a scheme of otherness ineunts-exeunts, the collection of all the collections, not having by definition any exeunt, is unthinkable. Cantor's paradox mathematically confirms this evidence that Peirce first attains through the categorical and semiotic status of individual. Real continuity is beyond any calculation that set theory can reach.

So what is this 'real' or 'perfect' continuity, which is beyond the 'pseudo-continuity' that sets can reach? Peirce changed his mind many times on this issue, trying first to tie continuity to necessity and then to possibility. Peirce first thought of making continuity the complete evolution of reality, the perfect 'generality' in his logical terms. In this version (1900-05), any singularity is a rupture of perfect continuity. But afterwards (1907-14) he connected continuity to a more complex pattern in which continuity is a possibility, namely a model that may be realized. Singularities are now realizations of that original possibility tending to a general cohesiveness (Peirce 1931-35: vol. 4, § 642).

It is in this second sense that our conception of change must be seen. We can understand change as a perfect continuity of possibilities of which any actual occurrence is a realization. Continuity is a law (general) whose internal regularity is 'an immediate connection' that we can understand as the condition of every possible realization (possibility). We can define a Peircean perfect continuum by four characters: modality (plasticity), transitivity, generality, and reflexivity, each underlying one aspect of the relationship between the parts and the whole of continuity, as seen in Zalamea 2001. Generality is the law of cohesiveness among parts beyond any individual and any possibility of metrically measuring it; modality means plasticity, namely the fact that a continuum is not tied to actualities but involves both possibility and necessity; transitivity is the internal passage between modalities (possibility, actuality, and general necessity); reflexivity means that any part shall have the same properties of the whole to which it belongs.

According to Peirce's 'extreme' realism, continuity coincides with reality and, thus, it founds mathematics. Since we discover continuity at the 'end' of inquiry on sets, it is an a posteriori foundation that happens while we are 'doing mathematics' through our scribing graphs and diagrams whether on the sheet of the mind or on some physical sheet. Mathematical diagrams work because they act synthetically, namely - according to an old Kantian definition - in mathematics we are dealing with universals in particulars, while in philosophy we have to deal with universals abstracted from particulars. The great power of generalization of mathematics is due to these contracted universals. We will call 'mathematical gesture' this kind of synthetic approach to mathematics through 'doing.'

23 There is a second interrelated approach to change in Peirce's late writings. It is the approach through logical modalities as such. Possibility, actuality, necessity, became the way in which Peirce explained transition within the continuum itself. If Cantor's paradox showed the existence of an original higher continuum of reality that exceeds our computations, modalities define the internal life of this continuity. But in what do these modalities consist? Possibility is the mode of reality in which the principle of contradiction does not hold. Actuality or existence is the mode of reality in which both the principle of contradiction and excluded third hold. Necessity is the mode of reality in 
which the principle of excluded third does not hold, that is, both alternatives can be false. Peirce's short version of the discovered ontological stands of modalities sums them up as 'may be's' (possibility), 'actualities,' and 'would be's' (necessity or generality). All three modalities are 'real' - meaning that they are independent from what any number of minds can think - or rather they coincide with the evolving continuity of reality. Reality actualizes possibilities and tries to develop them as generalities. According to Peirce, it is impossible to describe reality without modalities.

The logic of modalities implies a different understanding of stechiology (doctrine of elements). A term can be vague, determinate, and general (Peirce 1998: 350-3) according to the characters inhering in it. If any character is predicated universally and affirmatively or recognized as inherent the term is determinate. If it requires further determination by the utterer, it is vague; if it requires further determination by the interpretant it is general. Vagueness, determination, and generality fall under the same description of the logical modalities. What we have here is a transition in determination which is based on the ontological reality of the logical modalities. A term or a concept can be vague and become determinate and even general (and vice versa).

Vagueness has such an importance because it is the possible state of things from which ideas stem. Possibility as firstness can also be understood as a not yet actualized border from where actions (secondness) and ideas (thirdness) eventually emerge. The horotics of knowledge cannot thus dispense the complete triad vagueness/incarnation/abstraction. Moreover, it is precisely in the horotic transposition between particulars (seconds) and generals (thirds) where many logical forces enter the panorama. Creativity spurs from initial vagueness and many correlations between imagination (first) and reason (third) can be then precisely stated along the synthetic/horotic paradigm.

Logical features respect the four characteristics of the continuum: reflexivity, generality, possibility, transitivity. Every element has the same properties of general modalities (reflexivity); the passage among vagueness, determination, and generality (transitivity) is the law of the development of meaning through categories (generality); this latter depends on the possible inchoative status of vague meaning (possibility). Now, mathematical and logical characteristics of continuity showed what change is, and we are ready to explain what we mean by 'recognizing an identity through changes,' our definition of synthetic reasoning.

For our explanation we will rely on Peirce's Existential Graphs (hereafter EG), his iconicbased logics. EG were the way in which Peirce himself sought to represent continuity, and it is possibly one of the few scientific strategies of getting to the structure of 'recognizing' (see Roberts 1973). EG are the iconic formalization of logic of propositions (Alpha), first order (Beta), and modalities (Gamma). In comparison to formalization by symbols that happened in the same years, EG display a greater simplicity and uniformity (same rules for the three fragments, propositional, first order, modal). EG were still analytic, but their overall project was a synthetic one (Zalamea 2010). Using the phrase introduced above, they are a kind of 'mathematical gesture' and from this point of view they are synthetically conveying universals into particulars.

The synthetic 'doing' of the EG permits 'evidence' and 'generality.' Both properties are fundamental in Peirce's understanding of mathematics; and semiotics gave him the tools to understand their actual working. 'Evidence' is due to the iconic property of the graphs. Icons give to diagrams the fundamental visual character that makes them 'graspable' through perception. 'Generality,' is due to the so called 'hypostatic abstraction' that 
enables us to consider as object, and thus as diagram, any quality or aspect of reality. This kind of abstraction is the logical tool through which we pass "from 'good' to 'goodness' and the like" (Peirce 1998: 270n). The combination of the properties of 'evidence' and 'generality' accounts for our capacity of reading a diagram in a general way and to understand its relations. Generalization happens in 'doing' or 'scribing' those diagrams. If the generalization is the analytic result of the diagrams, diagrams are the synthetic happening of generals. 'Recognizing' is part of this synthetic 'happening' and should have the same evidence and generalization.

Recognizing always implies an object: 'recognizing something'; and the easiest way to look at it is to look at the case of identity. Here we will focus on an identity that is not a plain correspondence $\mathrm{A}=\mathrm{A}$, which according to Peirce himself is only a degenerate form of the real identity $\mathrm{A}=\mathrm{B}$. Identities are always passing through changes. $\mathrm{A}=\mathrm{A}$ is the static correspondence drawn from the set-theoretical definition of multitudes and it is a simplification of a 'more primitive' form of relation (Peirce 1976: vol. 4, 325-8).

Given our diagrams with their power of evidence and generalization, how can they help us understand our 'recognizing an identity'? In order to answer this question we have to represent both the continuity of change and the identity we want to inquire within this change. The first kind of continuity, the continuity of reality, is represented by the sheet of assertion. The sheet represents the universe of discourse we are dealing with in Alpha and Beta parts (logic of propositions and first order). But in the Gamma graphs (modal logic), when we want to represent change through modalities, we need a different continuum that 'must clearly have more dimensions' (Peirce 1931-35: vol. 4, § 512). This plastic multidimensional continuum is more 'original,' and the sheet of assertion of the Alpha and Beta parts is only a picture of this original continuum. In this continuum we are really scribing not the propositions as they actually are, but as they might be. We could think of them as marks or qualities, which cannot be numerable anymore - they are not assertions anymore - and they will exceed any multitude, according to our definition of 'perfect continuity'. This multidimensional continuum is apt to represent time or 'Becoming' (Peirce 1976: vol. 4, 330).

31 Now that we have the tool for representing the continuum of change, we can look for identity. In the Beta Graphs we have a graphical tool for identity: the line of identity, that can solve the problem of quantifiers. The line represents the existential quantifier when it is evenly enclosed and the universal quantifier through an appropriate nested double cut. But the definition becomes more complex when we do not have to deal with the two dimensional sheet of assertion, but with the multidimensional plastic continuum. When we scribe a line of identity on the multidimensional plastic continuum we are representing the identity into the continuum of possibilities. The line of identity continues to assert the identity of the individuals denoted by its extremities, but here it means the continuity of possibilities of an individual considered as a changing object in its becoming, i.e. the winner of Leipzig and the loser of Waterloo. This switch from using a line of identity on a sheet of assertion to using it on a multidimensional continuum implies the passage from an Assertoric view of Truth to a broader conception of truth and identity based on possibility. Identity is not anymore $\mathrm{A}=\mathrm{A}$ but a non-purely-symbolizable iconic identity passing from $\mathrm{A}$ to $\mathrm{B}$.

This switch also implies that the line of identity when it is scribed on a multidimensional continuum must become a line of teridentity, namely a line in which one of the extremities is a loose end. Teridentity represents two relations of co-identity and means 
that there are two different relations of identity that have in common one end and part of the line. The loose end of the line of teridentity means that there is or was also a different possibility even if it is or was not being actualized. It means that we know identity as a continuity but we do not know at which point it would stop and the point might be or have been different from the one we have. We have to allow the possibility that brute existence will be different since we are not representing actuality but potentiality. Finally, the line of identity has a direction or an aim because we draw it starting from one point and ceasing at another.

This is the tool Peirce crafted to define a changing identity analytically, but in order to grasp the synthetic view of identity, it is not EG's logical analytic use that is needed. The interesting features of the line of identity and teridentity come from Peirce's comment on the nature and the meaning of it: the line of identity, and a fortiori the line of teridentity, is another 'perfect continuum' along with the multidimensional continuum of assertion and, thus, it has the same properties we ascribed to continuity.

34 The line of identity is made of icons interpreted, and in so far is made of possibilities whose realizations are connected by a general rule (possibility and generality). The loose end guarantees the fullness of reflexivity: any part of the line, included the ends, are possibilities that might be realized according to a general law. Besides, every single dot actualized on the multidimensional continuum realizes a possibility according to the general law, and might have a different branch - the loose end - as a different actualization of the same possibility. So there is a transition from possibility to necessity through actuality. The translation of modalities in terms of vagueness, determination, and generalization is easy to see: identity is a progressive determination of possible qualities tending toward a generalization (tending to identify forever the winner of Leipzig and the loser of Waterloo) during the development (the 'becoming') expressed by the continuum within which they are inscribed.

How can this representation of identity be also the representation of 'recognizing an identity'? Peirce could unify 'identity' and 'recognizing an identity' by virtue of the practical 'scribing' on the sheet of assertion or on the multidimensional continuum. 'Recognizing an identity' in EG means to draw a line of identity between two points, knowing that there is always a different, loose possibility. The drawing itself is our recognizing; in $E G$ there is no recognition without the actual drawing of the line. It can be a mental or a virtual act, but it has to be a kind of diagram within a general interpretation. It is what we call a mathematical gesture.

What is a gesture? Gesture is any performed act which carries a meaning (from gero $=$ to bear, to carry). We can say we really understood something when we are ready to act according to our ideas; so, it is the performance that synthetically involves our reasoning. But if every gesture carries meaning, not every gesture serves to recognize an identity. So, any gesture is meaningful, but not every gesture embodies a synthetic reasoning. In order to understand 'syntheticity' we have to look for a 'perfect or complete gesture,' a gesture that respects all the characters we found following EG.

We should call a 'perfect or complete gesture' a gesture which has all the semiotic elements blended together almost equally. Now, for a gesture to be perfect it has to be a general law (symbol) that generates replicas; it is actual when it indicates its particular object (index); and it expresses different possibilities of aspects of the object which it refers to (icon). The three semiotic characteristics describe what a perfect gesture is: creative because of possible aspects, singular in its individuality, recognizable for its conformity to 
an established pattern. Moreover, gestures carry a possible and vague meaning that progressively gets determinate till a new habit is established. So we can easily find in this movement generality, modality, and transitivity.

Our new paradigm implies that a synthetic judgment (and reasoning) is a judgment (and reasoning) that recognizes - with evidence - identity through changes. How can we perform this reasoning? We do that through perfect gestures, where creativity, particularity and generality overlap. Perfect gestures - like the line of identity in EG save identity through changes because of their continuous semiotic nature. We obtain analytic reasoning by differentiation and horotic reasoning through a pendular 'integral and differential calculus' (see section 5). Analytic reasoning loses the identity through changes because it is concerned with breaking up the identity, as its etymology suggests (from analyo, discomposing, breaking up). It is very profitable in many cases but perfectly hopeless in many of our everyday businesses, and sometimes also - like in the case of discoveries or assessments of meaning - in many of our scientific activities (see section 5 , for the case of Contemporary mathematics). A horotic reasoning does not recognize an identity, but it is probably the richer state of our knowledge, the one in which our primeval beliefs lie.

\section{The Paradigm Along Gödel's Work}

Gödel's work is an outstanding example of the production of a great logician/ mathematician/philosopher, being able to explore in depth the full triad analytics/ synthetics/horotics. If his analytic power has been celebrated many times, his synthetic vision has been less so, in spite of his lifelong interest for intuitionistic logic (from Gödel 1932 up to Gödel 1972), his unusual physical imagination (Gödel 1949, 1952) and his enormous output of philosophical reflections (most still unpublished, beyond the 'standard' compilation Collected Works III; for an original, 'non standard' approach, see Cassou-Noguès 2007). Gödel's comprehensive and correlative interest in logic, mathematics and philosophy rests in fact on a synthetic understanding of knowledge. Moreover, in fairly detailed moments of Gödel's work one can disclose strong horotic forces, as we will indicate in this section.

In one of his philosophical discussions, Gödel proposes a 'general schema of possible philosophical world-views' which proves fruitful 'for the analysis of philosophical doctrines admissible in special contexts, in that one either arranges them in this manner or, in mixed cases, seeks out their materialistic and spiritualistic elements' (Gödel 1961: 375, our emphasis). Here, a synthetic perspective ('schema,' 'world-views') is presented first, and then applied to the analysis of philosophical doctrines along pragmatic situations ('special contexts,' 'mixed cases'). A pendulous horotics between matter and spirit governs the schema, and it is precisely in the border, mixed cases where the schema comes to be the most fruitful. Further, if we see that 'skepticism, materialism and positivism stand on one side, spiritualism, idealism and theology on the other,' Gödel affirms that 'it is a familiar fact, even a platitude, that the development of philosophy since the Renaissance has by and large gone from right to left - not in a straight line, but with reverses, yet still, on the whole' (Gödel 1961: 375, our emphasis; 'right'/spiritualism, 'left'/ materialism). The pendulum, with its back-and-forth horotics, is unavoidable. For Gödel, the deep mathematical concepts lie on right, against Hilbert's formalism tending to the left. Then, beyond the 'Hilbertian combination of materialism and aspects of classical 
mathematics' (Gödel 1961: 381), a 'workable combination' (Gödel 1961: 383), which reflects the idea that 'truth lies in the middle' (Gödel 1961: 381), should be proposed. Surprisingly, Gödel finds that such a middle horotics should be founded on Husserl's phenomenology, in order to capture an in-depth perception of mathematical objects. Thus Gödel comes very close to Peirce's ideas, as we have presented them in the previous sections, even if Gödel probably never knew Peirce's phaneroscopy and philosophy of mathematics (in Gödel's published work, there is only one reference to Peirce, on his calculus of relations (Gödel 1944: 120)).

On the technical side, all Gödel's papers on intuitionism include outstanding examples of synthetic and horotic strategies. His very first paper on intuitionism (Gödel 1932) exhibits already a precise synthetic property (non existence of a finite model which would capture provability of the intuitionistic calculus Int), and a precise horotic one (existence of infinite systems between intuitionistic and classical propositional calculi). Gödel 1933a extends, to first order, part of Glivenko's translatability criterion between Int and the classical propositional calculus, presenting, first, an intuitionistic Herbrand system $H^{\prime}$ with number variables (synthetic construction with enough signs for the semantics), and, second, a translation of the classical properties of partial recursive functions into $H^{\prime}$ (horotic transformation of calculability into intuitionistic provability). Gödel 1933b shows a new translation (horotic transformation) between Int and modal $S 4$, and conjectures their equiconsistency (full synthetic comparison). Finally, Gödel's famous Dialectica paper (Gödel 1958) presents a translation of provability in Heyting Arithmetic into computability of finite type functionals. Both the fairly detailed arithmetic systems (synthetics) and the translations (horotics) in play, acquire there in-depth stratified refinements.

One can understand Gödel as a thinker extremely sensible both to the recto and verso of a given situation (not only mathematical: his biography is full of such borderline sensitivities). We contend here that it is precisely thanks to this horotic temperament that Gödel's genius may have emerged. The presence of important horotic lines in Gödel's work can also in fact be signaled in what may be his most celebrated logical results. On one side, if, in his doctoral thesis, Gödel proves completeness of classical first-order logic, just one year later he discovers the incompleteness of Peano Arithmetic. The thin line where the tendency to insure completeness breaks (following Post, Presburger, Herbrand and Gödel himself) is a frontier that could only be conceived by an unusual mathematician, open both to the borders of proofs and to a via negativa approach to knowledge. Beyond Herbrand's bounded quantification, Gödel discovers the exact border - full induction - where incompleteness phenomena begin to occur. On the other side, if with the constructible universe Gödel proves the relative consistency of the Continuum Hypothesis $(\mathrm{CH})$, in his later years he proceeds again 'through the looking glass' and postulates natural axioms that force $c=X_{2}$, negating thus $(\mathrm{CH})$. All these examples in Gödel's work - philosophical and mathematical at large, logically detailed in his intuitionistic contributions - show perhaps the pertinence of our newly defined analytics/synthetics polarity and his natural extension to horotics. 


\section{The Paradigm Along Contemporary Developments of Mathematics}

The xxth Century French School in Philosophy of Mathematics (with names such as Poincaré, Brunschvicg, Cavaillès, Lautman, Desanti, Vuillemin, Châtelet, Petitot, Badiou) has stressed the central role of mathematical gestures for a fair understanding of mathematical creativity. Mathematical practice, contrasted with logical architecture, has to deal permanently with blind spots, obstructions, inconsistencies, contradictions. In such a perilous mathematical activity, often paths are explored through structural equilibrium or esthetic awareness (Shelah considers 'beauty' as the main reason for studying Set Theory), before rearranging the envisioned ideas on a sound logical basis. In these endeavors, both synthetic and horotic perspectives are fundamental.

Albert Lautman, possibly the most original philosopher of Modern mathematics (1830-1950), had stressed five main characteristics of mathematical 'higher' creativity (Galois, Riemann, Hilbert, E. Cartan, mainly studied by Lautman): (i) complex hierarchy of mathematical theories, irreducible with respect to intermediate deductive systems, (ii) semantic richness, irreducible to syntactical considerations, (iii) unity of structural methods behind the previous multiplicity, (iv) dynamics of mathematical practice/ gesture, (v) theorematic back-and-forth between unity and multiplicity (Lautman 2005). Lautman, a good friend of Charles Ehresmann, had anticipated the growing emergence of Category Theory (see, in particular, his brilliant analysis of the local/global polarity before the emergence of Sheaf Theory (Lautman 2010)), and our synthetic/horotic paradigm can also be traced back to Lautman's work. The global view fostered by (i) and (ii) require in fact synthetic approaches, while the bordering/dialectical view underlined in (iii)-(v) deals directly with an horotic approximation to knowledge. The dynamic structuralism in Philosophy of Mathematics proposed some decades ago (Awodey, Hellman, see Shapiro 2005), fully developed in the 1930's by Lautman, is thus much closer to a synthetic/horotic (mathematical) paradigm than to the analytic (logical) paradigm which encompassed the main 'Philosophy of Mathematics' (in fact, 'Philosophy of Logic') schools in the xxth century.

Extending Lautman's project to Contemporary mathematics (1950-today), we have underlined (Zalamea 2008) some features of contemporary mathematical creativity where the synthetic/horotic viewpoint helps to organize better the panorama: (vi) arithmetical structural impurity (Langlands, Deligne, Wiles, etc.), (vii) systematical geometrization (sheaves, co-homologies, geometric logic, etc.), (viii) freeness and schematization (groupoids, categories, topoi, motives, etc.), (ix) fluxion and deformation (non linearity, non commutativity, non elementarity, quantization, etc.), (x) reflexivity (classification, monster model, fixed points, etc.) In the amazingly productive period since 1950 (following Dieudonné, $99 \%$ of our actual mathematical knowledge would have therein emerged), the mathematical practice permanently breaks all analytic borders, merging together very diverse subfields of mathematics. Synthetic mixing is a source of inventiveness, and the horotic crossing of problems and ideas from different fields is a source of freshness for the discipline. appearances of synthetic and horotic forces vertebrate Grothendieck's work - his 
schemes, topoi or motives, crucially influential for Contemporary mathematics - one can also measure the importance of the synthetic/horotic paradigm for a fair understanding of the Philosophy of Mathematics. Grothendieck 1960-67 unifies and synthesizes, in the schemes, Riemann's vision (understanding of a complex curve through the ring of meromorphic functions over the curve) and Galois/Dedekind's vision (understanding of an algebraic variety through the spectrum of its maximal ideals). A horotic transposition helps to capture together, in a general setting, the ideas of non ramification and local/ global gluing (through sheaves on the spectrum of prime ideals). Grothendieck 1960-69 axiomatizes, through topoi, the movements of relative mathematics, generalizes the notion of point, discovers new geometric invariants and constructs the correct (co)homologies which would eventually lead to the solution of Weil's conjectures. Combining a synthetic view (mathematics from the top) and horotic gestures (multitude of new definitions, theorems and examples), Grothendieck explores systematically the borders of mathematical regions. On the other hand, inventing motives, Grothendieck looks for archetypes which lie at 'the heart or soul' of mathematical thought (Grothendieck 1985: 45). Beyond the multiplicity of (co)homologies, motives look for an initial or generic unity behind the various algebraic attempts to capture the fundamental mathematical contradiction discreteness/ continuity.

Some basic trends in Contemporary mathematics can be described through an iterated horotics between forms of idealism and realism (for a detailed account, see (Zalamea 2008: 99-150)). Eidal mathematics (from eidos, idea, and idein, vision) encompasses many works which open new territories through very general ideas and strong visual capabilities (Serre, Langlands, Lawvere, Shelah can be seen as such visionaries, for example). Quiddital mathematics (from quidditas, 'what it is') combines the capacity to work with rare abstract machineries and concrete physical problems (as in the work of Atiyah, Lax, Connes or Kontsevich). Archeal mathematics (from arkhê, beginning, and arkhên, commanding) unravels archetypical invariants along the eidal/quiddital transit, such as Friedman's reverse mathematics, Freyd's allegories, Zilber's trichotomy (extended by Hrushovski) or Gromov's $h$-principle. In each case, the dynamical forces of Contemporary mathematics, always crossing frontiers, always contaminating its diverse techniques, reveal a horotic factum difficult to understand from the perspectives of Analytic Philosophy.

An example of such a crucial mathematical contamination is obtained around the double analytic/synthetic content of the notion of sheaf (one of the main conceptual and historical border lines between Modern and Contemporary mathematics). On an analytic approach, the sheaf glues a coherent covering of neighborhoods, and, on a synthetic perspective, it produces a section which adequately preserves local properties of projections and restrictions (see figure 1):

Figure 1. Sheaves from Complementary Perspectives

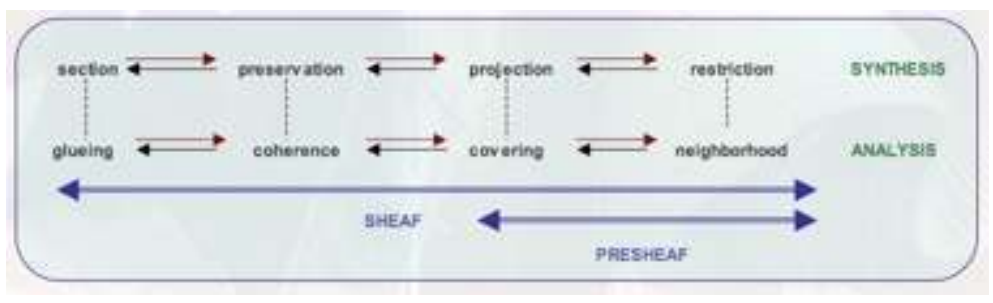



preservation-projection-restriction', we obtain a horotic transformation that we may call Grothendieck's Transform. In fact, the emergence of Grothendieck's topologies, at the beginning of Topos Theory, comes precisely from such a synthetic understanding of covers. But even better, Grothendieck's 'rising sea' technique is a profound methodological instance of that horotic transposition: to understand a mathematical concept/object (metaphorically a nut, with its hard shell), just cover it through diverse synthetic categories and functorial contexts (rising tides) until the concept is properly understood (the 'nourishing flesh' is then analytically uncovered, as the shell dissolves) (Grothendieck 1985: 552-3).

Grothendieck's Transform uses two distinct forms of understanding. Through transversality, it introduces a reference lattice where contrasts, coherence, asymptotic behavior and gluings can operate. In this way, for example, a non absolute, non analytic, notion of truth emerges, where a density of correct correlations stands as an approximation to truth, without invoking an ideal limit or a correspondence theory. On the other hand, through coverings, it introduces a dynamic fluidity, crucial for a sound comprehension of saturation processes in mathematics (a central characteristic first studied in all details by Lautman). Our epistemological perspectives change then radically, just by the simple fact of smoothing the situation: beyond polarities, the mediating transformations are integrated in an evolving fabric, closer to mathematical practice. Transversality in the work of Serre and in Langland's program, adjoint dialectics in Lawvere, tame coverings in Shelah's pcf theory, Atiyah's index theorem, harmonic analysis applied to the non euclidean wave equation in Lax's work, Freyd's intermediate categories, Zilber's proto-geometry, Gromov's $h$-principle, are very precise examples where transversality of coverings is fundamental. Since we are listing many great creative advances in Contemporary mathematics that cannot be understood from analytic perspectives, a natural space for a Synthetic/Horotic Philosophy of Mathematics emerges.

Beyond the usual ideal/real polarity, mathematical practice, particularly at the high level of Contemporary advances, is in fact producing an extremely interesting epistemological back-and-forth. The back-and-forth postulates not just a systematic oscillation between extremes, but one which is rooted on a coherent covering of the diverse partial approximations. Lindström's use of Cantor's back-and-forth in Abstract Model Theory is a paradigmatic use of the idea, but one can also detect it in Grothendieck's elucidation of the functorial properties of Teichmüller's space, in Shelah's structural amalgamation techniques, in Gromov's polynomial group growth, etc. All these processes reflect partial, hierarchical, distributed knowledge, which can be correctly understood only from perspectives open to transits along borders of mathematics, just what the synthetic/ horotic paradigm indicates.

\section{Forms of Synthetic/Horotic Reasoning for the XXIst Century}

Mathematical practice, as we have seen in previous sections - Peirce's mathematical gesture and his logic of continuity, Gödel's synthetic interplay between intuitionism and classical logic, contemporary mathematical developments along Grothendieck's legacy -

European Journal of Pragmatism and American Philosophy, IV-2 | 2012 
fosters a full triadic vision where analytic, synthetic and horotic techniques should complement each other. Nevertheless, from the viewpoint of Philosophy of Mathematics, the analytic preponderance in the xxth century has been overwhelming, and a balance is far from having been reached. In this final section we will (i) present the rough lines of how one should understand a Synthetic Philosophy, complementary to Analytic Philosophy, (ii) indicate a back-and-forth horotics between the two approaches, (iii) prospect a generalization of the situation for more extended cultural contexts, beyond mathematics.

53 A Synthetic Philosophy captures many ideas from what has been called 'Continental Philosophy,' but a systemic and systematic counterbalance with Analytic Philosophy can render clearer the panorama (see figure 2). Our main bet for the emergence and growth of such a Synthetic Philosophy may be summed up in the following diagram:

Figure 2. A New Paradigm for XxISt Century

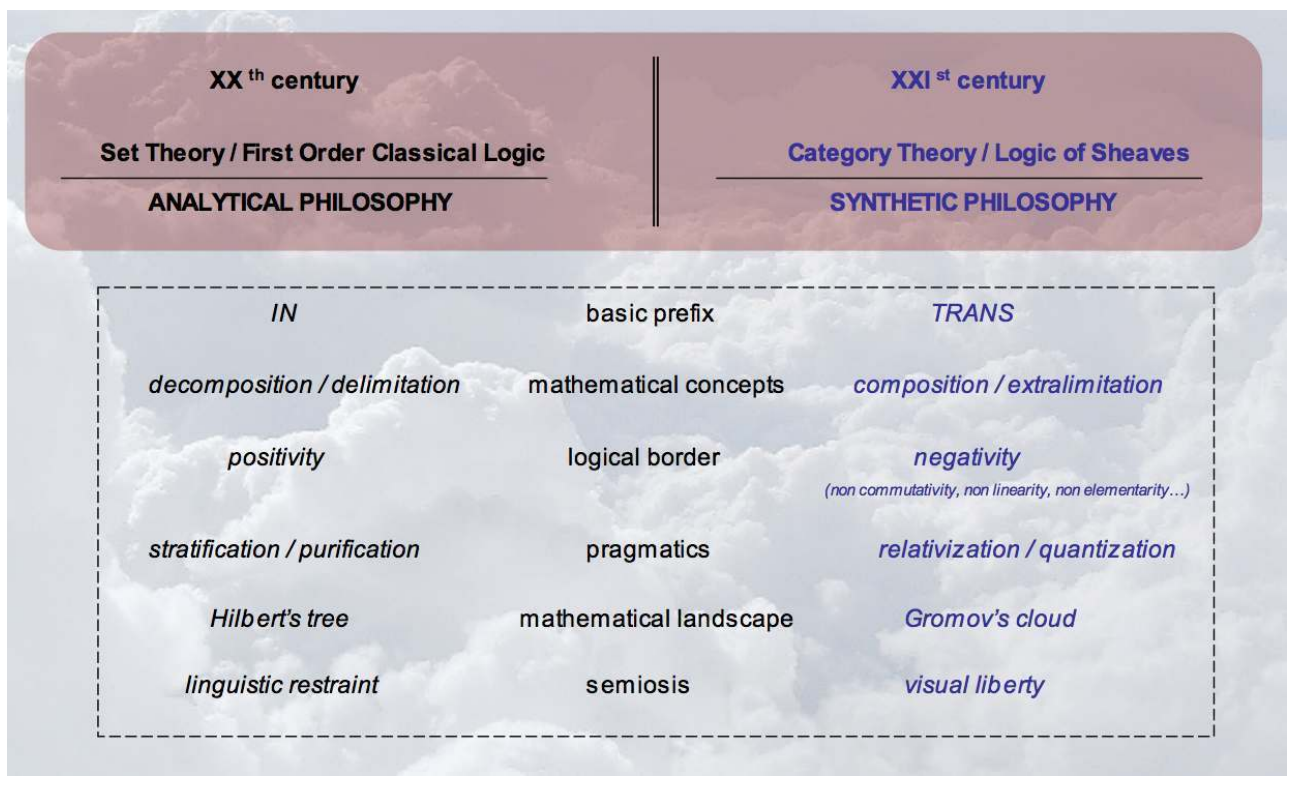

After due attention given to the right column in the preceding diagram, a more balanced understanding of the Philosophy of Mathematics should emerge in the next coming years. A full horotic back-and-forth between the two columns would then accommodate the guidelines of mathematical practice. In particular, the very complex 'Gromov's cloud' (Langevin 2000) - which tries to examine, under a new metaphor, exponential nodes, dense gluings, local wells, and all sort of multidimensional objects in Hilbert's mathematical tree - indicates that new geometric forms, particularly sensible to synthetic and horotic perspectives, are invading mathematical knowledge.

The philosophical generalization of the suggested scheme should show the breadth of its perspectives. Philosophy can find a new vein in the synthetic/horotic paradigm, eventually escaping the boundaries self-attributed according to the analytic pattern. We can easily observe that good philosophical fruits matured from the mathematical discoveries of the past two centuries. To them we owe the precision of the philosophy of language, the development of forms of artificial intelligence and their tools, good works in logic and their applications to informatics, a closer focus on single ethical, esthetical, and even ontological issues. As we pointed out, the analytic pattern from which all of that sprang involved a breaking down of problems to smaller and smaller theoretical pieces, in 
an endless run toward an impossible ultimate element. The new synthetic pattern built on 'recognizing an identity' will re-write epistemology, ethics, metaphysics, and pedagogy according to the same complementary attitude that we fostered all along in this paper. Semiotics and pragmatism will warrant the full horotic back and forth between the two patterns.

A few hints may suggest future ways of inquiry. In epistemology there are many topics that will have a different treatment and a complete list is impossible at this stage. But some topics are immediately questionable according to the synthetic/horotic pattern. First, we will look for a semiotic description of 'gestures' that can explain their working both analytically and synthetically. From Peirce's Existential Graphs we will be able to observe the semiotic structure of gestures understood as embodied in synthetic reasoning. When the pattern is established from a philosophical perspective, it is important to verify its plausibility in relationship with the actual development of mathematics. Second, many epistemological fields that did not find a complete explanation in the analytic pattern can be faced now through a synthetic view of reasoning relying upon semiotic based gestures. Among these unsolved topics, it is worth recalling: $i$ ) reference theory - so far blocked in the sterile alternative between descriptivism and causalism; ii) the theory of hypotheses - often mistaken as a psychological attitude; iii) creative processes, that have to be understood from both a logical and ontological perspective; iv) personal identity, which is directly connected with the ratio of synthetic reasoning as 'recognizing an identity'; $v$ ) the theory of the development of language, which can now be comprehended from the outset as a synthetic phenomenon of meaning, in transition from vagueness to generality. Third, our proposed synthetic/horotic pattern can change the approach to ethical and pedagogical issues. From the personal identity issue sprouts a different conception of ethics which has to be studied and developed. Gestures can encompass and overtake the alternative between a narrative historical pattern for ethics and a deontological one. An ethics founded on gestures can account for the continuity of moral personality and for our embodying rules and norms in a technical way, thanks to the mathematical ground and the semiotic structure of gestures. Finally, a pedagogy based on gestures should deepen the insight of Dewey's theory of education, which intended to mingle theory and practice, problems and solutions, precision of technical teaching and broad views of the unity of wisdom. Synthetic/horotic reasoning will overcome the fatal distinction between human and natural sciences that has determined a specialization without creativity and a creation without precise method.

\section{BIBLIOGRAPHY}

CASSOU-NOGUÈs P., (2007), Les démons de Gödel, Paris, Seuil.

GöDEL K., (1932), “On the Intuitionistic Propositional Calculus," in K. Gödel, Collected Works I: 222-5, Oxford, Oxford University Press [1986]. 
GÖDEL K., (1933a), “On Intuitionistic Arithmetic and Number Theory," in K. Gödel, Collected Works I: 286-95, Oxford, Oxford University Press [1986].

GÖDEL K., (1933b), “An Interpretation of the Intuitionistic Propositional Calculus,” in K. Gödel, Collected Works I: 300-3, Oxford, Oxford University Press [1986].

GÖDEL K., (1944), "Russell's Mathematical Logic," in K. Gödel, Collected Works II: 119-41, Oxford, Oxford University Press [1990].

GÖDEL K., (1949), “An Example of a New Type of Cosmological Solutions of Einstein's Field Equations of Gravitation," in K. Gödel, Collected Works II: 190-8, Oxford, Oxford University Press, [1990].

GÖDEL K., (1952), “Rotating Universes in General Relativity Theory," in K. Gödel, Collected Works II: 208-16, Oxford, Oxford University Press [1990].

GÖDEL K., (1958), “On a Hitherto Unutilized eExtension of the Finitary Standpoint,” in K. Gödel, Collected Works II: 240-51, Oxford, Oxford University Press [1990].

GÖDEL K., (1961), “The Modern Development of the Foundations of Mathematics in the Light of Philosophy," in K. Gödel, Collected Works III: 374-87, Oxford, Oxford University Press [1995].

GÖDEL K., (1972), "On an Extension of Finitary Mathematics which has not yet been used," in K. Gödel, Collected Works II: 271-80, Oxford, Oxford University Press [1990].

GROTHENDIECK A., (1960-67), Éléments de géometrie algébrique, (with J. Dieudonné), Paris, IHES.

GROTHENDIECK A., (1960-69), Séminaire de géometrie algébrique du Bois-Marie, (with diverse coauthors), Berlin, Springer [1970].

GROTHENDIECK A., (1985), “Récoltes et semailles,” unpublished manuscript.

KANT I., (1781/87), Critique of Pure Reason, Unified Edition, Indianapolis, Hackett Publishing Company [1996].

LANGEVIN R., (2000), “Interview: Mikhael Gromov," in J. P. Pier (ed.), Development of Mathematics 1950-2000, Boston, Birkäuser.

LAUTMAN A., (2005), Les idées, les mathématiques et le réel physique, Paris, Vrin.

LAUTMAN A., (2010 [1935]), "Rapport sur les travaux philosophiques entrepris par M. Lautman," Philosophiques 37, 9-15.

MADDALENA G., (2009), Metafisica per assurdo, Soveria Mannelli, Rubbettino.

PEIRCE C. S., (1931-35), Collected Papers, vols. I-VI, P. Weiss \& C. Hartshorne (eds.), Cambridge, Harvard University Press.

PEIRCE C. S., (1976), The New Elements of Mathematics, C. Eisele (ed.), The Hague, Mouton.

PEIRCE C. S., (1998), The Essential Peirce, vol. 2, ed. Peirce Edition Project, BloomingtonIndianapolis: Indiana University Press.

PEIRCE C. S., (1981-2010), Writings of Charles Sanders Peirce, vols. 1-6, 8, ed. Peirce Edition Project, Bloomington-Indianapolis, Indiana University Press.

ROBERTS D. D., (1973), The Existential Graphs of Charles S. Peirce, The Hague, Mouton.

SHAPIRO S. (2005), The Oxford Handbook of Philosophy of Mathematics and Logic, Oxford, Oxford University Press. 
ZALAMEA F., (2001), El continuo peirceano, Bogotá, Universidad Nacional de Colombia.

ZALAMEA F., (2008), Filosofía sintética de las matemáticas contemporáneas, Bogotá, Universidad

Nacional de Colombia (translation to appear: Falmouth, Urbanomic, 2012).

ZALAMEA F., (2010), Los gráficos existenciales peirceanos, Bogotá, Universidad Nacional de Colombia.

\section{NOTES}

1. Since 2001, through diverse books and articles, we had been wandering around the ideas of frontier, border, thirdness and vagueness, in order to get a natural completion of the dyad analytics/synthetics, when Roberto Perry offered us (2009) the perfect, unspoiled, term: horotics. We gratefully acknowledge his support.

\section{ABSTRACTS}

We study a contemporary need to complement analytic philosophy with pendular, synthetic approaches. We provide new definitions of the dyad analytics/synthetics and complete it with a natural third, horotics. Some historical trends to support a synthetic/horotic paradigm are studied: (i) Peirce's ideas around his logic of continuity - non Cantorian continuum and existential graphs - emphasizing the importance of mathematical gestures, (ii) Gödel's understanding of intuitionism as a synthetic counterpart of classical logic, along with a new horotic approach to his work, (iii) Contemporary mathematical achievements (1950-2000), difficult to understand from analytical philosophy perspectives. Finally, we indicate some main features that a systematical synthetic/horotic reasoning should enforce, in order to fulfill its historic sequence.

\section{AUTHORS}

\section{GIOVANNI MADDALENA}

Università del Molise

gmaddal3[at]hotmail.com

\section{FERNANDO ZALAMEA}

Universidad Nacional, Colombia

fernandozalamea[at]gmail.com 